\title{
Optimization of p-type contacts to InGaN-based laser diodes and light emitting diodes grown by plasma assisted molecular beam epitaxy
}

\author{
KrZesimir NOWAKOWSKI-SZKUDLAREK*, GrZEGORZ MUZIOL, Mikolaj ŻAK, \\ Mateusz HAJDEL, MarCin SIEKACZ, ANNA FEDUNIEWICZ-ŻMUDA, \\ CZESLAW SKIERBISZEWSKI \\ Institute of High Pressure Physics PAS, Sokołowska 29/37, 01-142 Warsaw, Poland \\ ${ }^{*}$ Corresponding author: szkudlarek@unipress.waw.pl
}

\begin{abstract}
We investigated the influence of the $\operatorname{In}_{0.17} \mathrm{Ga}_{0.83} \mathrm{~N}: \mathrm{Mg}$ contact layer grown by plasma assisted molecular beam epitaxy on the resistivity of p-type $\mathrm{Ni} / \mathrm{Au}$ contacts. We demonstrate that the Schottky barrier width for p-type contact is less than $5 \mathrm{~nm}$. We compare circular transmission line measurements with a p-n diode current-voltage characteristics and show that discrepancies between these two methods can occur if surface quality is deteriorated. It is found that the most efficient contacts to p-type material consist of $\operatorname{In}_{0.17} \mathrm{Ga}_{0.83} \mathrm{~N}: \mathrm{Mg}$ contact layer with $\mathrm{Mg}$ doping level as high as $2 \times 10^{20} \mathrm{~cm}^{-3}$.
\end{abstract}

Keywords: gallium nitride, molecular beam epitaxy, contacts.

\section{Introduction}

The p-type contacts to nitride based light emitting diodes (LEDs) and laser diodes (LDs) is one of the key issues for increasing the wall-plug-efficiency of these devices. The wide bandgap for nitrides creates the basic disadvantage for making ohmic contacts to p-type region - namely, the lack of the metal with the Fermi level position located close to the III-N valence band maximum. Therefore all existing contacts to p-III-N suffer from the existence of the Schottky barrier which increases the voltage drop of the device. Therefore, the engineering of the proximity of the semiconductor/metal interface is crucial for decreasing the turn-on voltage and contact resistance. There are several literature reports on the influence of contact layer and metallization $[\underline{1}, \underline{2}]$ on the contact resistance for devices grown by metal-organic vapor phase epitaxy (MOVPE). There are different strategies for making this proximity surface layer applied to improve contact properties like very heavy GaN:Mg doping or growth of InGaN:Mg contact layer [2] .

However, in a case of plasma assisted molecular beam epitaxy (PAMBE) the way of preparation of good ohmic p-type contacts is less investigated [2- $\underline{5}$ ]. The recent advancement in the PAMBE in the fabrication of III-nitride LDs [] ], especially the demonstration of LDs lifetime of 100000 hours [7], was accompanied with optimization of contacts 
to p-type layers. This was especially important, since for hydrogen free PAMBE process, the location of the Fermi level position can be different than one for MOVPE. Among other solutions we successfully applied the approach based on the InGaN:Mg contact layer [2] .

In this work we describe the influence of the InGaN:Mg layer thickness and $\mathrm{Mg}$ doping level on the resistivity of the metal $(\mathrm{Ni} / \mathrm{Au})$ contacts in vertical and horizontal configuration. Two measurement configurations are used: $i$ ) circular transmission line measurements (CTLM) and $i i)$ p-n diode current-voltage ( $I-V)$ characteristics. Contradictory results are found with these two methods. We propose a simple explanation based on deterioration of surface quality and the appearance of grain boundaries within the InGaN:Mg contact layer.

\section{Experimental methods}

All samples presented in this work were grown in VG V90 MBE reactor equipped with two Veeco RF-plasma (radio frequency plasma) sources. The InGaN (at indium-rich condition) and $\mathrm{AlGaN}$ (at gallium-rich condition) layers were grown at $650^{\circ} \mathrm{C}$. The metal -rich conditions were used to ensure step flow growth and smooth surface at low temperatures. However, for a heavily doped InGaN contact layer we found deterioration of the crystalline quality which can be attributed to the influence of high $\mathrm{Mg}$ doping. The sample structure is shown in Fig. 1. Details of growth mechanism in PAMBE can be found elsewhere [ $[$ ] .

As it was reported in many works, the main role in the reduction of p-type contact resistance plays the contact layer [ $\underline{2}-\underline{6}]$. This layer should be Mg doped as high as possible due to high ionization energy of $\mathrm{Mg}$ (only $1 \%$ of $\mathrm{Mg}$ is ionized at room temperature). However, there are at least two effects which can prevent high acceptor doping. First of all, for very high $\mathrm{Mg}$ doping levels (above $2 \times 10^{19} \mathrm{~cm}^{-3}$ ), it is known that an auto-compensation of $\mathrm{Mg}$ occurs, which reduces p-type conductivity (probably doubly

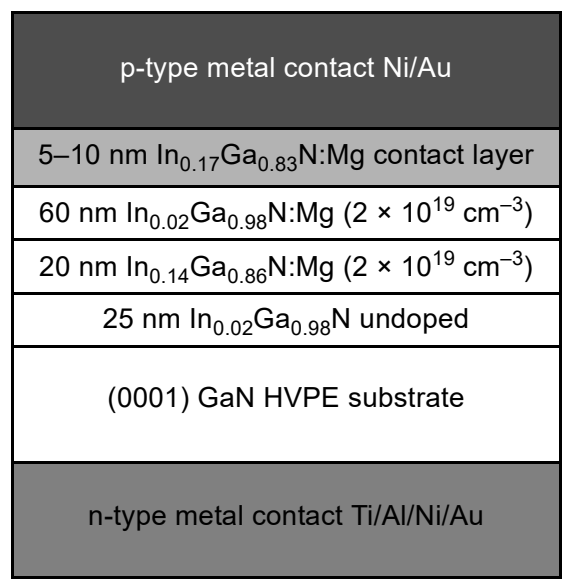

Fig. 1. Details of samples grown by PAMBE. 
T a b l e. The details of the sample contact layer grown by PAMBE.

\begin{tabular}{lcllll}
\hline $\begin{array}{l}\text { Sample } \\
\text { number }\end{array}$ & $\begin{array}{l}\mathrm{In}_{0.17} \mathrm{Ga}_{0.83} \mathrm{~N} \\
\text { contact layer } \\
\text { thickness }[\mathrm{nm}]\end{array}$ & $\begin{array}{l}\text { Mg doping } \\
\text { level }\left[\mathrm{cm}^{-3}\right]\end{array}$ & $\begin{array}{l}\text { Contact resistivity } \\
\text { from CTLM } \\
{\left[\Omega \mathrm{cm}^{2}\right]}\end{array}$ & $\begin{array}{l}\text { Layer resistivity } \\
\text { from CTLM } \\
{[\Omega / \square]}\end{array}$ & $\begin{array}{l}\text { Voltage } \\
\text { at } 2 \mathrm{kA} / \mathrm{cm}^{2} \\
\text { from } I-V[\mathrm{~V}]\end{array}$ \\
\hline$\# 1(\mathrm{REF})$ & 5 & $1 \times 10^{20}$ & $1.0 \times 10^{-2}$ & $7.3 \times 10^{4}$ & 4.06 \\
$\# 2$ & 10 & $1 \times 10^{20}$ & $8.6 \times 10^{-2}$ & $1.7 \times 10^{4}$ & 4.02 \\
$\# 3$ & 5 & $2 \times 10^{20}$ & $4.6 \times 10^{-3}$ & $3.9 \times 10^{4}$ & 3.75 \\
$\# 4$ & 5 & $5 \times 10^{19}$ & $1.4 \times 10^{-3}$ & $1.9 \times 10^{4}$ & 4.07 \\
\hline
\end{tabular}

ionized Mg-related donors are created) [ $\underline{8}, \underline{9}]$. Also, for high $\mathrm{Mg}$ flux, when accumulation of $\mathrm{Mg}$ on a grown surface occurs, it can lead to the polarity inversion (PI) [10]. After the PI, the Mg incorporation efficiency drops by two orders of magnitude leading to very poor p-type conductivity [10]. These effects were found important for growth of thick p-type layers. It is still unknown, how growth of very thin (few monolayers) InGaN: $\mathrm{Mg}$ is affected by $\mathrm{Mg}$ auto-compensation or PI. For instance, for our samples, even for very high $\mathrm{Mg}$ doping levels (as high as $2 \times 10^{20} \mathrm{~cm}^{-3}$ ), we do not observe PI. Since the PI reverts Ga polarity to N polarity, it is sensitive to the etching in $\mathrm{KOH}$. We found that our samples were resistant to such etching. On the other hand, the contact layer morphology became rough, and we observe that heavy doping can create a number of defects and grain boundaries in contact layers. The Table contains the description of samples, in which we changed only the parameters of contact layer.

The doping efficiency of standard Mg $k$-cell (Knudsen cell-type of effusion evaporator source) is limited and it is very difficult to change Mg flux easily by an order of magnitude as it is required for contact layer. However, the $\mathrm{Mg}$ doping level is inversely proportional to the growth rate of $\mathrm{InGaN}$ at low growth temperatures $\left(650^{\circ} \mathrm{C}\right)$. To increase the doping level in the $\operatorname{In}_{0.17} \mathrm{Ga}_{0.83} \mathrm{~N}$ contact layer, we reduced its growth rate 7 times from 0.36 to $0.05 \mu \mathrm{m} / \mathrm{h}$ (expecting that the $\mathrm{Mg}$ doping will increase by the same value). It was achieved by reduction of the Ga flux. It is known that the growth rate $G_{\mathrm{R}}$ of InGaN depends on the Ga flux $\Phi_{\mathrm{Ga}}$ and the $\operatorname{In}_{x} \mathrm{Ga}_{1-x} \mathrm{~N}$ composition $x$ : $G_{\mathrm{R}}=\Phi_{\mathrm{Ga}} /(1-x)$ [11]. To introduce in more detail the way in which we achieved heavily doped contact layer, we will concentrate on characteristics of InGaN growth. In Fig. 2 we present the dependence of the InGaN composition (Fig. 2a) and the growth rate (Fig. 2b) as a function of $\Phi_{\mathrm{Ga}}$. The solid lines presented in Fig. 2 are based on a theoretical model which accounts for InGaN growth (for nonequivalent atomic step edges) [11]. This model is well proved by experimental data [11]. Points A and B show the growth conditions for growth of $\operatorname{In}_{0.17} \mathrm{Ga}_{0.83} \mathrm{~N}: \mathrm{Mg}$ contact layer and $\operatorname{In}_{0.02} \mathrm{Ga}_{0.98} \mathrm{~N}: \mathrm{Mg}$ layer located below (see Fig. 1). For both layers the $\mathrm{Mg}$ flux from $k$-cell was the same. Since there is a deterioration of the surface quality of heavily doped layers, the analysis of SIMS data on the reference InGaN:Mg layer is challenging: it shows, e.g., smearing of the $\mathrm{Mg}$ profile. However, the value of the $\mathrm{Mg}$ incorporation from SIMS data $\left(2 \times 10^{20} \mathrm{~cm}^{-3}\right.$ for reference layer to sample \#3) agrees with the calculations based on the difference of the growth rate and known values of $\mathrm{Mg}$ incorporation to InGaN layers for high growth rate with good crystal quality. 

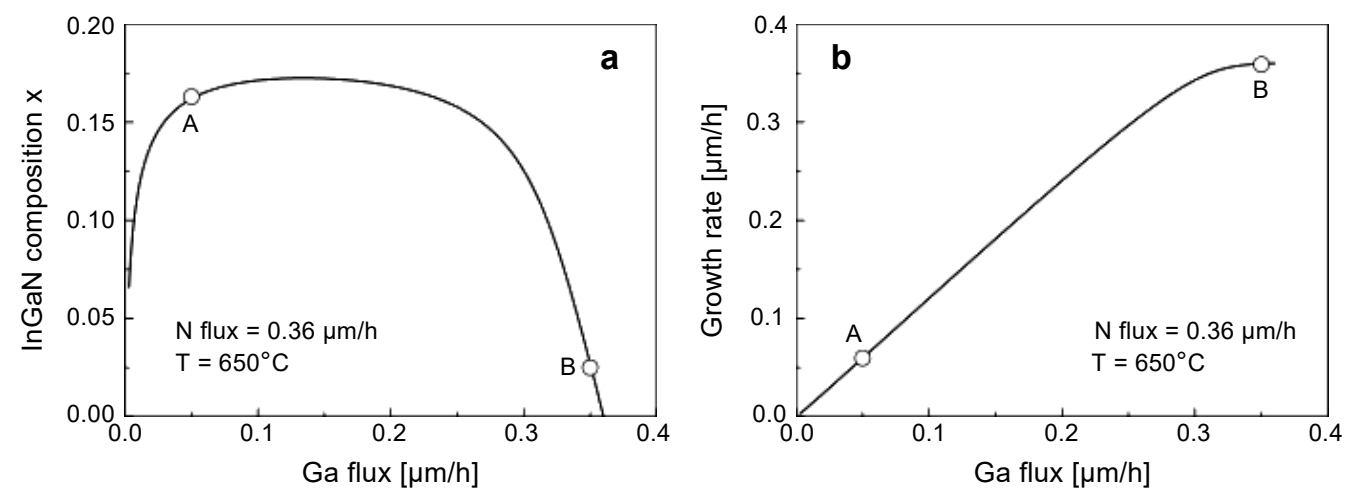

Fig. 2. The dependence of InGaN composition (a) and the growth rate (b) on Ga flux for the InGaN layers grown by PAMBE: $\mathrm{A}-\mathrm{In}_{0.17} \mathrm{Ga}_{0.83} \mathrm{~N}$ contact layer, $\mathrm{B}-\mathrm{In}_{0.02} \mathrm{Ga}_{0.98} \mathrm{~N}$ layer.

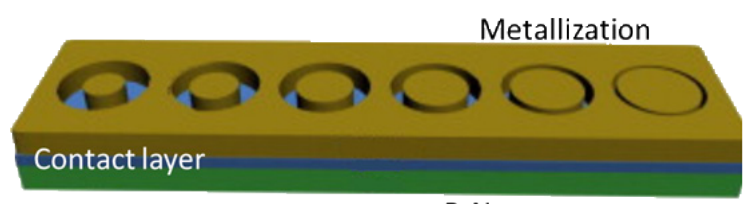

$$
\text { P-N }
$$

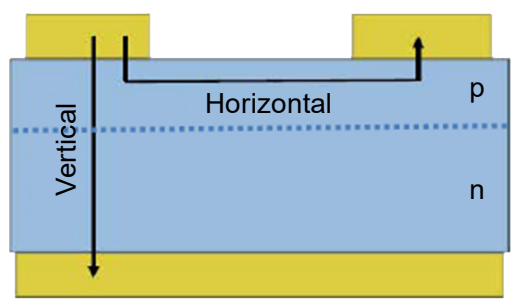

b

Fig. 3. Birds-eye view of the metal geometry on the samples (a). Cross-section schematic showing the current paths in two measurement configurations (b).

For electrical measurements, we processed p-type contacts appropriate for CTLM (Fig. 3). In addition, the bottom n-type contact was formed, which allows to perform the vertical $I-V$ characterization of the p-n diode like for standard LEDs.

For the p-type metal contacts we used $25 \mathrm{~nm}$ Ni followed by $75 \mathrm{~nm}$ of Au which were annealed at $500^{\circ} \mathrm{C}$ for 10 minutes in oxygen atmosphere with rapid thermal annealing (RTP). The n-type contact was formed as a sequence of $\mathrm{Ti}(30 \mathrm{~nm}), \mathrm{Al}(60 \mathrm{~nm})$, $\mathrm{Ni}(80 \mathrm{~nm})$ and $\mathrm{Au}(150 \mathrm{~nm})$. Contacts for all samples were made during one run.

\section{Results and discussion}

\subsection{Morphology of grown samples}

The atomic force microscopy (AFM) investigations of our samples reveal that InGaN:Mg contact layer is very rough. We do not observe clear atomic steps on the surface, which 


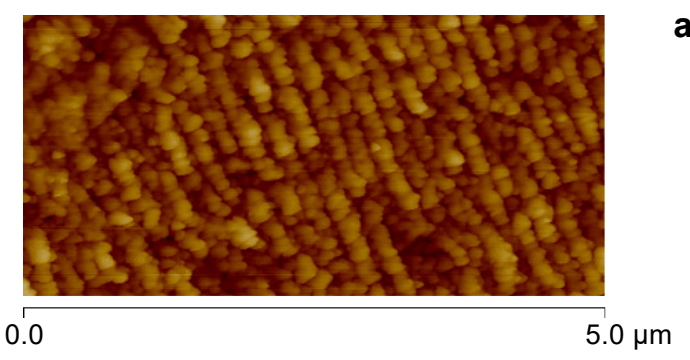

a

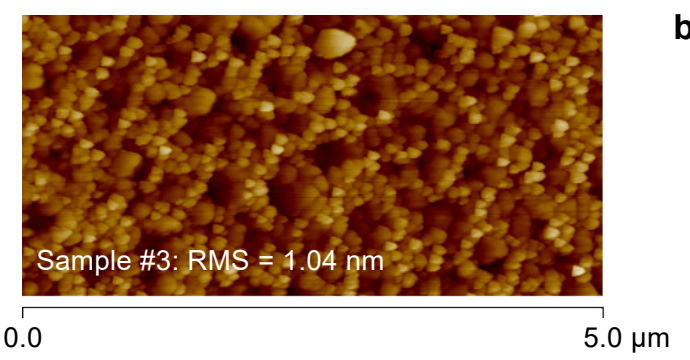

Fig. 4. The AFM images of the samples: \#4 (a) and \#3 (b).

we normally observe on undoped or lightly doped layers [12]. Instead, island nucleation is observed. In addition, roughness increases when we increase the Mg doping level. This trend is clearly seen when we compare sample \#4 with root mean square RMS = $=1.04 \mathrm{~nm}$ (Fig. 4a) and sample \#3 with RMS =0.80 nm (Fig. 4b). We will come back to this result in the discussion of the electric properties of our samples.

\subsection{Electrical properties}

The most interesting data come when we compare the vertical and horizontal conductivity of our samples. Horizontal transport was measured using CTLM (Figs. 3 and 5a).
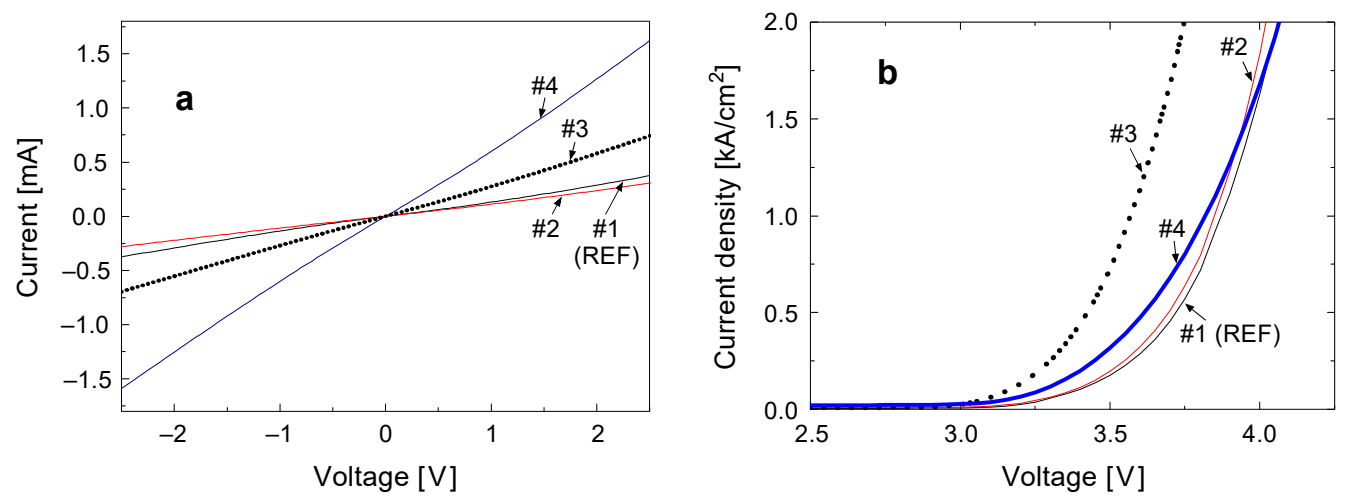

Fig. 5. CTLM characteristics (for $80 \mu \mathrm{m}$ inner contact size, $20 \mu \mathrm{m}$ ring spacing between contacts - horizontal configuration) (a) and p-n diode $I-V$ characteristics $(100 \mu \mathrm{m} \times 100 \mu \mathrm{m}$ contact size - vertical configuration) of samples with different contact layers (b). 
It allowed to define the resistance of InGaN contact layer and the resistance of the metal contact to the semiconductor. We also investigated the p-n diode $I-V$ characteristics in vertical configuration (Fig. 5b). The results obtained from the analysis of CTLM and $\mathrm{p}$-n diode $I-V$ data are gathered in the Table. First of all, we would like to comment the behavior of samples \#1 and \#2. The increase of the thickness of the contact layer from 5 to almost $10 \mathrm{~nm}$ does not change contact layer conductivity and $I-V$ characteristics (in comparison with samples \#3 and \#4). We interpret this result as a proof that the width of Schottky barrier between our InGaN contact layer and $\mathrm{Ni} / \mathrm{Au}$ metal is lower than $5 \mathrm{~nm}$. If Schottky barrier between InGaN contact layer and $\mathrm{Ni} / \mathrm{Au}$ metal was higher than $5 \mathrm{~nm}$, then the decrease in the resistance of metal contact to the semiconductor for the sample with $10 \mathrm{~nm}$ InGaN contact layer would be observed.

When we changed the doping level of the contact layer (samples \#1, \#3 and \#4) we observed strange results from CTLM experiments. All samples presented in Fig. 5a have ohmic contacts. From CTLM experiments the best sample is \#4, i.e. with the lowest doping level in contact layer. Sample \#4 has the highest currents at the same voltages and the lowest resistance metal contact to the semiconductor. Sample \#4 also shows the best morphology - see AFM picture (Fig. 4a). On the other hand, from the analysis of $I-V$ characteristics, the lowest voltage is obtained using the highest $\mathrm{Mg}$ doping level (sample \#3). This inconsistency in experimental data can be explained in the following way. The heavy doping of contact layer leads to deterioration of layer quality, as we can observe from the AFM data. The formation of islands influences transport properties of measured layers. However, only the horizontal configuration (CTLM) is affected. The current flow in the horizontal configuration suffers from a higher resistivity if the InGaN:Mg contact layer is not rigid (Fig. 6). On the other hand, current flow in the vertical configuration (Fig. 6) is not influenced by the island formation. Therefore, the key element which enabled us to understand the behavior of samples \#3 and \#4 is the morphology of the contact layers. As we already noticed, the morphology for sample \#3 is rougher than for sample \#4. This means that for sample \#3, the horizontal transport is highly influenced by the number of grain boundaries and therefore we observe a higher resistivity of this layer. On the other hand, this sample may have a lower

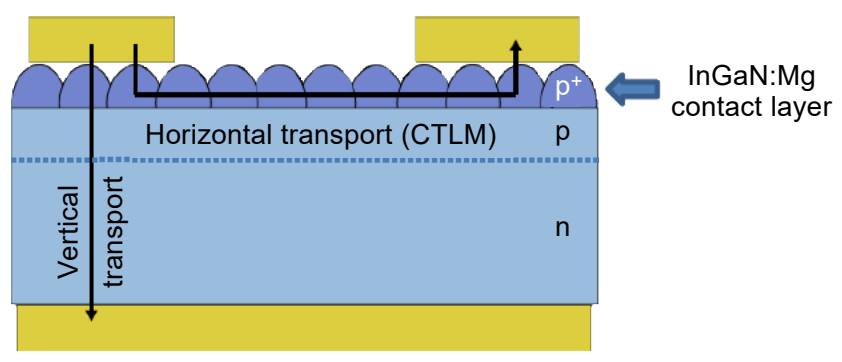

Fig. 6. Horizontal and vertical transport with $\mathrm{In}_{0.17} \mathrm{Ga}_{0.83} \mathrm{~N}$ contact layer. Horizontal transport is influenced by scattering on grain boundaries. 
depletion width of the Schottky barrier due to higher doping and therefore a lower voltage in the vertical $I-V$ characteristics.

\section{Conclusions}

We studied the properties of InGaN:Mg for efficient p-type contact layer in optoelectronic devices like LEDs and LDs grown by PAMBE. The practical way how to increase the doping level in InGaN layers by reduction of the growth rate is proposed. We demonstrated that for our $\operatorname{In}_{0.17} \mathrm{Ga}_{0.83} \mathrm{~N}: \mathrm{Mg}$ contact layer/Ni/Au metal contact, the Schottky barrier width is less than $5 \mathrm{~nm}$. The lowest operating voltage was achieved for the highest Mg doping level of contact layer. However, the CTLM data brought contradictory results. We attribute this discrepancy to additional resistance due to grain boundaries which were created in heavily doped samples. Therefore, the optimization of contact layer relying solely on CTLM (or transmission line measurements (TLM)) experiments might bring false conclusions.

Acknowledgements - This work was supported by TEAM-TECH POIR.04.04.00-00-210C/16-00 Project of the Foundation for Polish Science co-financed by the European Union under the European Regional Development Fund and the National Centre for Research and Development grant LIDER/35/0127/L-9/ $17 / \mathrm{NCBR} / 2018$.

\section{References}

[1] Kumakura K., Makimoto T., Kobayashi N., Low-resistance nonalloyed ohmic contact to p-type GaN using strained InGaN contact layer, Applied Physics Letters 79(16), 2001, pp. 2588-2590, DOI: 10.1063/1.1410336.

[2] Greco G., Iucolano F., Roccaforte F., Ohmic contacts to gallium nitride materials, Applied Surface Science 383, 2016, pp. 324-345, DOI: 10.1016/j.apsusc.2016.04.016.

[3] TANG H., SAdaf S. M., Wu X., JiAng W., Highly efficient p-type doping of GaN under nitrogen-rich and low-temperature conditions by plasma-assisted molecular beam epitaxy, AIP Advances 9(5), 2019, article 055008, DOI: 10.1063/1.5089658.

[4] Yoo M.C., Lee J.W., Myoung J.M., Shim K.H., Kim K., Low resistance ohmic contact on p-type GaN grown by plasma-assisted molecular beam epitaxy, MRS Proceedings 423, 1996, pp. 131-136, DOI: 10.1557/proc-423-131.

[5] Pearton S.J., Abernathy C.R., Ren F., Gallium Nitride Processing for Electronics, Sensors and Spintronics, Springer, London, 2006, DOI: 10.1007/1-84628-359-0.

[6] Skierbiszewski C., Turski H., Muziol G., Siekacz M., Sawicka M., Cywiński G., Wasilewski Z.R., PoROWSKI S., Nitride-based laser diodes grown by plasma-assisted molecular beam epitaxy, Journal of Physics D: Applied Physics 47(7), 2014, article 073001, DOI: 10.1088/0022-3727/47/7/073001.

[7] Muziol G., Siekacz M., Nowakowski-Szkudlarek K., Hajdel M., Smalc-Koziorowska J., Feduniewicz -Żmuda A., Grzanka E., Wolny P., Turski H., Wiśniewski P., Perlin P., Skierbiszewski C., Extremely long lifetime of III-nitride laser diodes grown by plasma assisted molecular beam epitaxy, Materials Science in Semiconductor Processing 91, 2019, pp. 387-391, DOI: 10.1016/j.mssp.2018.12.011.

[8] Iida D., Tamura K., Iwaya M., Kamiyama S., Amano H., Akasaki I., Compensation effect of Mg-doped a- and c-plane GaN films grown by metalorganic vapor phase epitaxy, Journal of Crystal Growth 312(21), 2010, pp. 3131-3135, DOI: 10.1016/j.jcrysgro.2010.07.038. 
[9] Castiglia A., Carlin J.-F., Grandjean N., Role of stable and metastable Mg-H complexes in p-type GaN for cw blue laser diodes, Applied Physics Letters 98(21), 2011, article 213505, DOI: 10.1063/ 1.3593964.

[10] Feduniewicz A., Skierbiszewski C., Siekacz M., Wasilewski Z.R., Sproule I., GrZanka S., Jakiela R., Borysiuk J., Kamler G., Litwin-Staszewska E., Czernecki R., Boćkowski M.,Porowski S., Control of Mg doping of GaN in RF-plasma molecular beam epitaxy, Journal of Crystal Growth 278(1-4), 2005, pp. 443-448, DOI: 10.1016/j.jcrysgro.2005.01.004.

[11] Turski H., Siekacz M., Wasilewski Z.R., Sawicka M., Porowski S., Skierbiszewski C., Nonequivalent atomic step edges-Role of gallium and nitrogen atoms in the growth of In GaN layers, Journal of Crystal Growth 367, 2013, pp. 115-121, DOI: 10.1016/j.jcrysgro.2012.12.026.

[12] Skierbiszewski C., Siekacz M., Perlin P., Feduniewicz-Zmuda A., Cywiński G., Grzegory I., LeszczyŃski M., Wasilewski Z.R., Porowski S., Role of dislocation-free GaN substrates in the growth of indium containing optoelectronic structures by plasma-assisted MBE, Journal of Crystal Growth 305(2), 2007, pp. 346-354, DOI: 10.1016/j.jcrysgro.2007.04.002.

Received October 16, 2019 in revised form January 8, 2020 